\title{
A Finite Element Subproblem Method for Position Change Conductor Systems
}

\author{
Patrick Dular ${ }^{1,2}$, Laurent Krähenbühl ${ }^{3}$, Ruth V. Sabariego ${ }^{1}$, Mauricio V. Ferreira da $\mathrm{Luz}^{4}$, \\ Patrick Kuo-Peng ${ }^{4}$ and Christophe Geuzaine ${ }^{1}$ \\ ${ }^{1}$ University of Liège, Dept. of Electrical Engineering and Computer Science, ACE, B-4000 Liège, Belgium \\ ${ }^{2}$ F.R.S.-FNRS, Fonds de la Recherche Scientifique, B-1000 Brussels, Belgium \\ ${ }^{3}$ Université de Lyon, Ampère (CNRS UMR5005), École Centrale de Lyon, F-69134 Écully Cedex, France \\ ${ }^{4}$ GRUCAD/EEL/UFSC, Po. Box 476, 88040-970 Florianópolis, Santa Catarina, Brazil
}

\begin{abstract}
Analyses of magnetic circuits with position changes of both massive and stranded conductors are performed via a finite element subproblem method. A complete problem is split into subproblems associated with each conductor and the magnetic regions. Each complete solution is then expressed as the sum of subproblem solutions supported by different meshes. The subproblem procedure simplifies both meshing and solving processes, with no need of remeshing, and accurately quantifies the effect of the position changes of conductors on both local fields, e.g. skin and proximity effects, and global quantities, e.g. inductances and forces. Applications covering parameterized analyses on conductor positions to moving conductor systems benefit from the developed approach.
\end{abstract}

Index Terms-Finite element method (FEM), subdomain method, conductor systems.

\section{INTRODUCTION}

A subproblem method (SPM) with finite element (FE) solutions provides advantages in repetitive analyses and helps improving the solution accuracy [1]-[6]. It allows to benefit from previous computations instead of starting a new complete FE solution for any variation of geometrical or physical data. It also allows different problem-adapted meshes and computational efficiency due to the reduced size of each SP.

A FE-SPM is herein developed for coupling solutions of position change conductors in magnetic systems, with the aim to accurately calculate the changes of both local fields (skin and proximity effects, reaction fields, local forces) and global quantities (currents, voltages, inductances, Joule losses, forces). Both massive and stranded conductors are considered, in parameterized analyses on their positions, naturally extended to moving conductor systems.

The SPM combines any changes via volume sources (VSs), originated from previous solutions and applied via mesh-tomesh projections. The developments are performed for the magnetic vector potential FE magnetodynamic formulation, paying special attention to the proper discretization of the constraints involved in each SP and to the resulting weak FE formulations and circuit relations. The method is illustrated and validated on test problems.

\section{Coupled Magnetic SubProblems}

\section{A. Sequence of Subproblems}

Complete models are proposed to be split into sequences of SPs, gathering sets of conductors and magnetic regions. The SP solutions are to be added to give the complete solution.

Manuscript received July 1, 2011. Corresponding author: P. Dular (e-

Digital Object Identifier inserted by IEEE
This offers a way to perform parameterized analyses, with a direct access to each change. The parameters can be the positions of the conductors, as well as their conductivities.

Each SP is defined in its own domain. At the discrete level, this aims to decrease the problem complexity and to allow distinct meshes with suitable refinements and possible domain overlapping. No remeshing is necessary when adding a region or changing its position.

\section{B. Canonical magnetic problem}

A canonical 2-D or 3-D magnetodynamic problem $p$, to be solved at step $p$ of the SPM, is defined in a domain $\Omega_{p}$, with boundary $\partial \Omega_{p}=\Gamma_{p}=\Gamma_{h, p} \cup \Gamma_{b, p}$. The eddy current conducting part of $\Omega_{p}$ is denoted $\Omega_{c, p}$ and the non-conducting one $\Omega_{c, p}{ }^{C}$, with $\Omega_{p}=\Omega_{c, p} \cup \Omega_{c, p}{ }^{C}$. Massive conductors belong to $\Omega_{c, p}$, whereas stranded conductors belong to $\Omega_{s, p} \subset \Omega_{c, p}{ }^{C}$. The equations and material relations of problem $p$ are

$$
\begin{array}{rr}
\operatorname{curl} \boldsymbol{h}_{p}=\boldsymbol{j}_{p}, \operatorname{div} \boldsymbol{b}_{p}=0, \quad \operatorname{curl} \boldsymbol{e}_{p}=-\partial_{t} \boldsymbol{b}_{p}, \quad(1 \mathrm{a}-\mathrm{b}-\mathrm{c}) \\
\boldsymbol{h}_{p}=\mu_{p}^{-1} \boldsymbol{b}_{p}+\boldsymbol{h}_{s, p}, \quad \boldsymbol{j}_{p}=\sigma_{p} \boldsymbol{e}_{p}+\boldsymbol{j}_{s, p}, \quad(2 \mathrm{a}-\mathrm{b})
\end{array}
$$

where $\boldsymbol{h}_{p}$ is the magnetic field, $\boldsymbol{b}_{p}$ is the magnetic flux density, $\boldsymbol{e}_{p}$ is the electric field, $\boldsymbol{j}_{p}$ is the electric current density, $\mu_{p}$ is the magnetic permeability, $\sigma_{p}$ is the electric conductivity and $\boldsymbol{n}$ is the unit normal exterior to $\Omega_{p}$. Note that (1c) is only defined in $\Omega_{c, p}$ (as well as $\boldsymbol{e}_{p}$ ), whereas it is reduced to the form (1b) in $\Omega_{c, p}{ }^{C}$. Boundary conditions (BCs) on $\boldsymbol{n} \times \boldsymbol{h}_{p} \mid \Gamma_{h, p}$, $\left.\boldsymbol{n} \cdot \boldsymbol{b}_{p}\right|_{b, p}$ or $\boldsymbol{n} \times\left.\boldsymbol{e}_{p}\right|_{\Gamma_{e, p} \subset \Gamma_{b, p}}$ have to be defined, acting as surface sources (SSs) possibly expressed from previous solutions [2][6].

The fields $\boldsymbol{h}_{s, p}$ and $\boldsymbol{j}_{s, p}$ in (2a-b) are VSs. The source $\boldsymbol{h}_{s, p}$ is usually used for fixing a remnant induction. The source $\boldsymbol{j}_{s, p}$ fixes the current density in inductors. With the SPM, $\boldsymbol{h}_{s, p}$ is also used for expressing changes of permeability and $\boldsymbol{j}_{s, p}$ for changes of conductivity, or for adding portions of inductors [4]-[6]. For changes in a region, from $\mu_{q}$ and $\sigma_{q}$ for problem $q$ 
to $\mu_{p}$ and $\sigma_{p}$ for problem $p$, the associated VSs $\boldsymbol{h}_{s, p}$ and $\boldsymbol{j}_{s, p}$ are

$$
\boldsymbol{h}_{s, p}=\left(\mu_{p}^{-1}-\mu_{q}^{-1}\right) \boldsymbol{b}_{q}, \boldsymbol{j}_{s, p}=\left(\sigma_{p}-\sigma_{q}\right) \boldsymbol{e}_{q} .
$$

Each problem $p$ is constrained via the so defined VSs and SSs from parts of the solutions of other problems. This offers a wide variety of changes [2]-[6]. The complete solution is

$$
\boldsymbol{u}=\sum_{p \in P} \boldsymbol{u}_{p}, \text { with } \boldsymbol{u} \equiv \boldsymbol{h}, \boldsymbol{b}, \boldsymbol{j}, \boldsymbol{e}, \ldots
$$

with $P$ an ordered set of SPs. A correction can become a significant source for any of its source problems, which is inherent to large perturbation problems. In this case, an iterative process between the related SPs has to be done till convergence up to a desired accuracy [4]. In addition to the iterations between SPs, classical inter-problem iterations are needed in nonlinear analyses. Each solution $\mathbf{u}_{p}$ can then be calculated as a series of corrections $\mathbf{u}_{p, i}$ (with $i$ the sub-SP (SSP) index), i.e.

$$
\boldsymbol{u}_{p}=\sum_{i} \boldsymbol{u}_{p, i}=\boldsymbol{u}_{p, 1}+\boldsymbol{u}_{p, 2}+\ldots
$$

\section{Canonical b-Conform Weak Formulation}

Equations (1b-c) are fulfilled via the definition of a magnetic vector potential $\boldsymbol{a}_{p}$ and an electric scalar potential $v_{p}$,

$$
\operatorname{curl} \boldsymbol{a}_{p}=\boldsymbol{b}_{p}, \boldsymbol{e}_{p}=-\partial_{t} \boldsymbol{a}_{p}-\operatorname{grad} v_{p}, \text { with } \boldsymbol{u}_{p}=\operatorname{grad} v_{p} \cdot(6 \mathrm{a}-\mathrm{b}-\mathrm{c})
$$

The weak $\boldsymbol{b}$-conform $\boldsymbol{a}_{p}$-formulation of problem $p$ is given by the weak form of the Ampère equation (1a), i.e. [4],

$$
\begin{aligned}
& \left(\mu_{p}^{-1} \operatorname{curl} \boldsymbol{a}_{p}, \operatorname{curl} \boldsymbol{a}^{\prime}\right)_{\Omega_{p}}+\left(\boldsymbol{h}_{s, p}, \operatorname{curl} \boldsymbol{a}^{\prime}\right)_{\Omega_{p}}-\left(\boldsymbol{j}_{s, p}, \boldsymbol{a}^{\prime}\right)_{\Omega_{p}} \\
& +\left(\sigma_{p} \partial_{t} \boldsymbol{a}_{p}, \boldsymbol{a}^{\prime}\right)_{\Omega_{c, p}}+\left(\sigma_{p} \boldsymbol{u}_{p}, \boldsymbol{a}^{\prime}\right)_{\Omega_{c, p}}=0, \forall \boldsymbol{a}^{\prime} \in F_{p}^{1}\left(\Omega_{p}\right),
\end{aligned}
$$

where $F_{p}{ }^{1}\left(\Omega_{p}\right)$ is a curl-conform function space defined on $\Omega_{p}$, gauged in $\Omega_{c, p}{ }^{C}$, and containing the basis functions for $\boldsymbol{a}_{p}$ as well as for the test function $\boldsymbol{a}^{\prime}$ (at the discrete level, this space is defined by edge FEs; the gauge is based on the treeco-tree technique); $(\cdot, \cdot)_{\Omega}$ denotes a volume integral in $\Omega$ of the product of its vector field arguments.

\section{Mesh-to-Mesh Projection of VSs}

Some parts of a previous solution $\boldsymbol{a}_{q}$ serve as sources (VSs) in a subdomain $\Omega_{s, p} \subset \Omega_{p}$ of the current problem $p$. At the discrete level, this means that this source quantity $\boldsymbol{a}_{q}$ has to be expressed in the mesh of problem $p$, while initially given in the mesh of problem $q$. This is done via a projection method [7] of its curl limited to $\Omega_{s, p}$, i.e.

$$
\left(\operatorname{curl} a_{q-p}, \operatorname{curl} a^{\prime}\right)_{\Omega_{s, p}}=\left(\operatorname{curl} a_{q}, \operatorname{curl} a^{\prime}\right)_{\Omega_{s, p}}, \forall a^{\prime} \in F_{p}^{1}\left(\Omega_{s, p}\right),
$$

where $F_{p}{ }^{1}\left(\Omega_{s, p}\right)$ is a gauged curl-conform function space for the $p$-projected source $\boldsymbol{a}_{q-p}$ (the projection of $\boldsymbol{a}_{q}$ on mesh $p$ ) and the test function $\boldsymbol{a}^{\prime}$.

\section{CONDUCTORS IN PROBLEM SPLITTINGS}

\section{A. Massive Conductors}

The circuit relation of a massive conductor $\Omega_{c, p}$, relating its current $I_{p}$ and voltage $U_{p}$ (circulation of $-\boldsymbol{u}_{p}$ along the conductor) changes, due to contributions from a problem $q$ (possibly gathering several SP solutions), is obtained by using $\boldsymbol{a}^{\prime}=\operatorname{grad} v^{\prime}=\boldsymbol{u}^{\prime}$ as test function in (7) [9], i.e.

$$
\left(\sigma_{p} \partial_{t} \boldsymbol{a}_{p}, \boldsymbol{u}^{\prime}\right)_{\Omega_{c, p}}+\left(\sigma_{p} \boldsymbol{u}_{p}, \boldsymbol{u}^{\prime}\right)_{\Omega_{c, p}}-\left(\boldsymbol{j}_{s, p}, \boldsymbol{u}^{\prime}\right)_{\Omega_{c, p}}=I_{p}
$$

with $\boldsymbol{j}_{s, p}$ given by (3b) with (6b-c), i.e.

$$
\boldsymbol{j}_{s, p}=\left(\sigma_{p}-\sigma_{q}\right) \boldsymbol{e}_{q}=-\left(\sigma_{p}-\sigma_{q}\right)\left(\partial_{t} \boldsymbol{a}_{q}+\boldsymbol{u}_{q}\right) .
$$

Adding a massive conductor - For a newly added $\Omega_{c, p},(9)$ gives its initial current and voltage $\left(I_{p, 1}\right.$ and $\left.U_{p, 1}\right)$. These are accurately calculated, with actual skin and proximity effects, if problem $p$ is solved with (7) and (9) with sources from problem $q$. If solution $p$ is only initially known for $\Omega_{c, p}$ alone, with no contribution from solution $q$, only its own skin effect is then considered. This can give a good initial approximation of its circuit relation, that can be further improved with an additional component of solution $p$ with source $q$.

Changing a massive conductor-For an $\Omega_{c, p}$ already considered as a source of problem $q$, that added some regions, previous components of solution $p$ (e.g. $\boldsymbol{a}_{p, l}$ and $\boldsymbol{u}_{p, l}$ ) are known. The current iteration on problem $p$ aims at calculating this solution change (e.g. $\boldsymbol{a}_{p, 2}$ and $\boldsymbol{u}_{p, 2}$ ), which can be done at two levels. As a first approximation, if no current change is allowed $\left(I_{p, 2}=0\right)$ and proximity effects due to solution $q$ are neglected (thus with no need to solve (7)), $\boldsymbol{a}_{p, 2}=0$ and (9) simply leads to a voltage change, via $\boldsymbol{u}_{p(, 2)}$ (the SSP index is omitted to lighten the expressions) given by

$$
\left(\boldsymbol{u}_{p}, \boldsymbol{u}^{\prime}\right)_{\Omega_{c, p}}=-\left(\partial_{t} \boldsymbol{a}_{q}, \boldsymbol{u}^{\prime}\right)_{\Omega_{c, p}}
$$

For the accurate level considering proximity effects, $\boldsymbol{a}_{p}$ and $\boldsymbol{u}_{p}$ need to be solved with (7) and (9), usually with $\boldsymbol{h}_{s, p}=0$ and $\sigma_{q}=0$, i.e. (3b) reduced to $\boldsymbol{j}_{s, p}=\sigma_{p} \boldsymbol{e}_{q}$. This leads to the accurate circuit relation change.

\section{B. Stranded Conductors}

For a stranded conductor or coil $\Omega_{s, p}$, the circuit relation relating its current $I_{p}$ and voltage $U_{p}$ changes is

$$
\left(\partial_{t} \boldsymbol{a}_{p}, \boldsymbol{j}^{\prime}\right)_{\Omega_{s, p}}+\left(\partial_{t} \boldsymbol{a}_{q}, \boldsymbol{j}^{\prime}\right)_{\Omega_{s, p}}+R_{p} I_{p}=-U_{p},
$$

where $R_{p}$ is the coil resistance and $\boldsymbol{j}^{\prime}$ is a global test function defined for the considered coil as

$$
\boldsymbol{j}^{\prime}=\boldsymbol{j}_{S, p}=N_{S} / S_{S} \boldsymbol{t}
$$

with $N_{S}$ its number of turns, $S_{S}$ its total surface area and $\boldsymbol{t}$ the unit vector tangent to the coil direction [8], [9].

Adding a stranded conductor - If $\Omega_{s, p}$ is newly added, (12) relates its initial $I_{p, 1}$ and $U_{p, 1}$, including the effect of solution 
$q$ via the term $\left(\partial_{t} \boldsymbol{a}_{q}, \boldsymbol{j}_{s, p}\right)_{\Omega_{\mathrm{s}, \mathrm{p}}}$. Solving (7) with this conductor, together with (12), is required.

Changing a stranded conductor- If $\Omega_{s, p}$ was already considered as a source of problem $q$, previous components of solution $p$ (e.g. $\boldsymbol{a}_{p, 1}, U_{p, 1}$ and $I_{p, 1}$ ) are known. Changes of this solution $p$ are then given by (7) and (12), with again the need to calculate the contribution $\left(\partial_{t} \boldsymbol{a}_{q}, \boldsymbol{j}_{s, p}\right)_{\Omega_{\mathrm{s}, \mathrm{p}}}$ in (12).

If no current change is allowed in $\Omega_{s, p}$, i.e. $I_{p(2)}=0$, the associated solution of (7) is simply $\boldsymbol{a}_{p,(2)}=0$. Relation (12), with (13), is thus reduced to

$$
U_{p}=-\left(\partial_{t} a_{q}, j_{s, p}\right)_{\Omega_{s, p}},
$$

giving the voltage change $U_{p(, 2)}$ and consequently the inductance change of the coil.

The contribution in (12) from a previous solution $q$, i.e. $\left(\partial_{t} \boldsymbol{a}_{q}, \boldsymbol{j}_{s, p}\right)_{\Omega_{\mathrm{s}, \mathrm{p}}}$, gains to be evaluated indirectly from integrals on the modified regions that were sources of $\boldsymbol{a}_{q}$; this avoids any integration in $\Omega_{s, p}$ which would need to project $\boldsymbol{a}_{q}$ on its mesh. For this, in the SP sequence, one gets back to the previous iteration of problem $p$ preceding problem $q$ (or, in the newly added conductor case, one considers the actual problem $p$ ), and uses $\boldsymbol{a}_{q}$ and $\boldsymbol{a}_{p}$, respectively, as test functions in their formulations, i.e. (to lighten the development, one omits with no limitation the possible contributions of massive conductors)

$$
\begin{gathered}
\left(\mu_{p}^{-1} \operatorname{curl} \boldsymbol{a}_{p}, \operatorname{curl} \boldsymbol{a}_{q}\right)_{\Omega_{p}}+\left(\boldsymbol{h}_{s, p}, \operatorname{curl} \boldsymbol{a}_{q}\right)_{\Omega_{p}}-\left(\boldsymbol{j}_{s, p}, \boldsymbol{a}_{q}\right)_{\Omega_{p}}=0, \\
\left(\mu_{q}^{-1} \operatorname{curl} \boldsymbol{a}_{q}, \operatorname{curl} \boldsymbol{a}_{p}\right)_{\Omega_{q}}+\left(\boldsymbol{h}_{s, q}, \operatorname{curl} \boldsymbol{a}_{p}\right)_{\Omega_{q}}-\left(\boldsymbol{j}_{s, q}, \boldsymbol{a}_{p}\right)_{\Omega_{q}} \\
+\left(\sigma_{q} \partial_{t} \boldsymbol{a}_{q}, \boldsymbol{a}_{p}\right)_{\Omega_{c, q}}+\left(\sigma_{q} \boldsymbol{u}_{q}, \boldsymbol{a}_{p}\right)_{\Omega_{c, q}}=0
\end{gathered}
$$

Subtracting the resulting expressions, i.e. (15) to (16), with $\Omega_{p} \equiv \Omega_{q}, \boldsymbol{h}_{s, q}=\left(\mu_{q}^{-1}-\mu_{p}^{-1}\right) \boldsymbol{b}_{p}(3 \mathrm{a}), \boldsymbol{j}_{s, q}=-\left(\sigma_{q}-\sigma_{p}\right)\left(\partial_{t} \boldsymbol{a}_{p}+\boldsymbol{u}_{p}\right)$ (10) and usually $\boldsymbol{h}_{s, p}=0$ and $\sigma_{p}=0$, one has

$$
\begin{gathered}
\left.\left.\left(\boldsymbol{j}_{s, p}, \boldsymbol{a}_{q}\right)_{\Omega_{s, p}=-((} \mu_{q}^{-1}-\mu_{p}^{-1}\right)\left(\operatorname{curl} \boldsymbol{a}_{p}+\operatorname{curl} \boldsymbol{a}_{q}\right), \operatorname{curl} \boldsymbol{a}_{p}\right)_{\Omega_{p}} \\
+\left(\sigma_{q}\left(\partial_{t}\left(\boldsymbol{a}_{p}+\boldsymbol{a}_{q}\right)+\boldsymbol{u}_{q}\right), \boldsymbol{a}_{p}\right)_{\Omega_{c, p}} .
\end{gathered}
$$

Only some integrals on the modified regions of problem $q$ (where $\mu_{q}$ and $\mu_{p}$ differ) remain with the term $\left(\boldsymbol{j}_{s, p}, \boldsymbol{a}_{q}\right)_{\Omega_{\mathrm{s}, \mathrm{p}}}$, which is the time primitive of the term to be evaluated, thus via the other remaining integrals. This is a remarkable result that allows a very accurate calculation of the inductance change, in particular in non-destructive testing problems.

If the primary coil is excited with a fixed current $\left(I_{p, 1}\right)$, its proper field remains unchanged whatever the reaction field of the added regions. Its inductance change $L_{p, 2}$ is calculated with (14) and (17). If voltage excited, or excited via an external electric circuit, its inductance change gives a current correction $\left(I_{p, 2}\right)$. There is no need to solve again the correction primary coil problem. Its solution is simply obtained by weighting the primary solution $\left(\boldsymbol{a}_{p, 2}=\boldsymbol{a}_{p, 1} I_{p, 2} / I_{p, 1}\right)$. An iterative process, on the SSP indices, can be done between the primary coil problem and the added region problems.

\section{APPLICATION EXAMPLES}

To illustrate and validate the SPM, TEAM problem 17 is studied, dealing with a jumping ring [10]. This problem is well adapted to the SPM, allowing tests of progressive levels of difficulty, from magnetostatic to magnetodynamic problems, from frequency to time domain, from axisymmetric to 3-D models, from current to voltage sources, etc., also with moving bodies. Furthermore it needs accurate calculations of global quantities, e.g. self and mutual inductances and forces. The basic rig comprises an exciting coil plus a set of inner cores, various plates, and conducting rings. Progressive sets of experiments include the main coil in air, the main coil with laminated iron core, then above a large conducting plate, then with one ring added. A search coil can be added as well. These are axisymmetric, from magnetostatic to magnetodynamic, problems. They can go up to 3-D problems with other positions of the plates.

Adding a magnetic core - For a sinusoidal input current of $1 \mathrm{~A}$ at $50 \mathrm{~Hz}$ in the main coil (500 turns, inner radius $40 \mathrm{~mm}$, width $37.5 \mathrm{~mm}$ and height $100 \mathrm{~mm}$ ), the primary coil solution $\left(a_{1}\right)$ is a source for the reaction field of an added inner magnetic core $\left(\mu_{r}=300\right.$, height $150 \mathrm{~mm}$, radius $25 \mathrm{~mm}$, bottom $12 \mathrm{~mm}$ below the main coil), calculated as a correction solution $\left(\boldsymbol{a}_{2}\right)$ (Fig. 1). Two different meshes are used (Fig. 1). Adding these two solutions, the coil-core solution is obtained and checked to be similar to the complete solution. The coil reactance components are $\mathrm{X}_{1,1}=5.2 \Omega$ (calculated from (12)) and $\mathrm{X}_{1,2}=14.3 \Omega$ (calculated from (14) with (17)), of which the sum is $19.5 \Omega$, checked to be equal to the coil reactance for the complete problem.

Adding a massive conductor - For an interval of positions of an added massive conducting ring $\left(\sigma=2.0810^{7} \mathrm{~S} / \mathrm{m}\right.$, inner radius $38.5 \mathrm{~mm}$, width $6.35 \mathrm{~mm}$ and height $25.4 \mathrm{~mm}$ ) and the sinusoidal input current in the main coil, the eddy current densities in the ring (Fig. 2) and the ensuing forces are calculated. The magnetic core is present. This is done with the SPM, decoupling the meshes of the magnetic source (coil and magnetic core) and of the moving ring, and the classical approach with remeshing for any new position of the ring. From these results, Fig. 3 shows the height of the ring versus the input current. For a similar accuracy, a speed-up factor of about 100 is obtained with the SPM, thanks to the noremeshing and the reduction of the computational domain for each position change of the ring. The speed-up factor increases with the number of positions considered. The primary coil reactance change is again calculated with high accuracy, e.g. for the ring $12.5 \mathrm{~mm}$ above the main coil, $\mathrm{X}_{1,3}=-6.7 \Omega$ (calculated from (14) with (17)), giving for the total reactance $12.8 \Omega$ calculated for the complete problem. The ring resistance is also correctly calculated, $R_{3}=3.7 \Omega$, in perfect accordance with the complete problem.

Adding a stranded conductor - The accuracy obtained with the SPM approach on the calculation of the mutual inductance 
between the main coil and a search coil (50 turns, inner radius $100 \mathrm{~mm}$, width $50 \mathrm{~mm}$ and height $12 \mathrm{~mm}$ ) is pointed out in Fig. 4. A significant speed-up factor is again obtained with the SPM (about 120) in comparison with the classical approach.

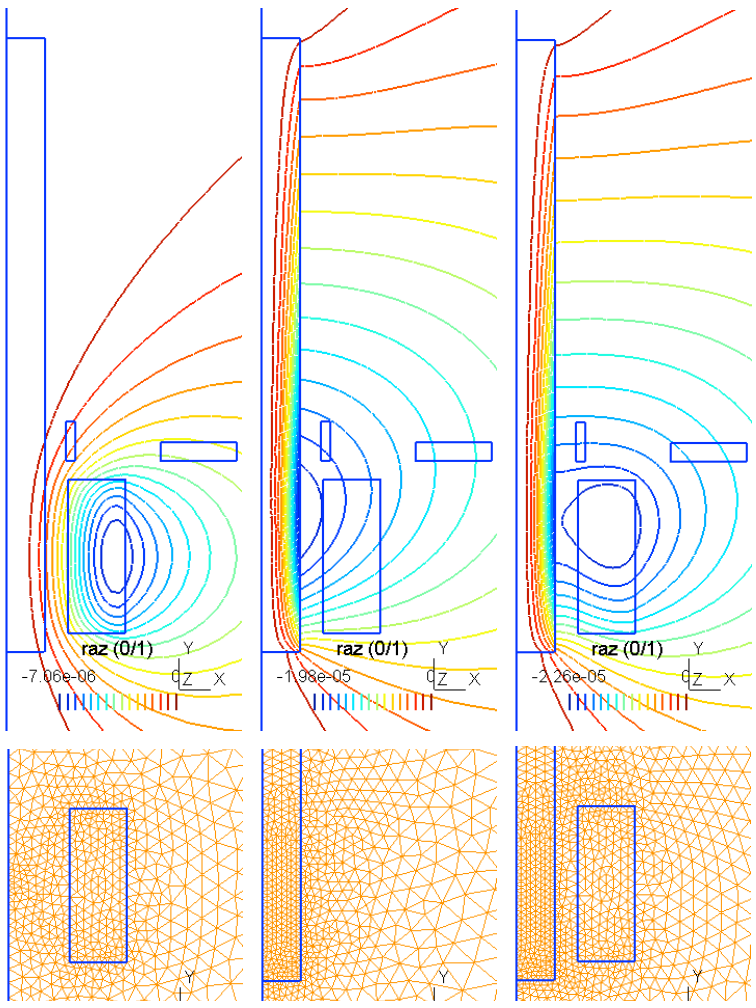

Fig. 1. Top: Field lines for the primary coil alone $\left(\boldsymbol{b}_{1}\right.$, left $)$, for the added inner core $\left(\boldsymbol{b}_{2}\right.$, middle $)$ and the total field $\left(\boldsymbol{b}=\boldsymbol{b}_{1}+\boldsymbol{b}_{2}\right.$, right $)$. Bottom: associated meshes. The conducting ring and the search coil are shown but are still inactive (i.e. not included in the meshes).
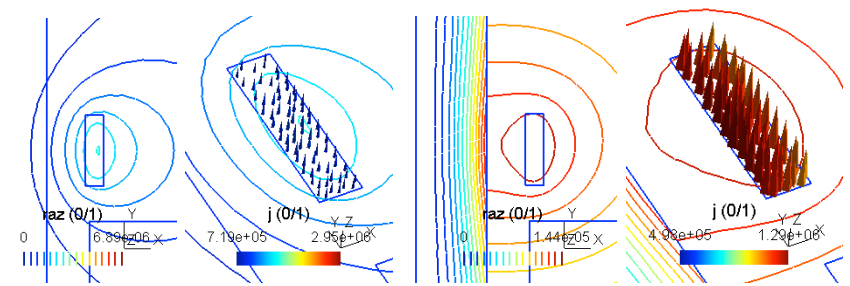

Fig. 2. Field lines $\left(\boldsymbol{b}_{3}\right)$ and eddy current density $\left(\boldsymbol{j}_{3}\right)$ in the added conducting ring, without (left; only $\boldsymbol{a}_{2,1}$ ) and with (right; up-dating $\boldsymbol{a}_{2}$ and $\boldsymbol{a}_{3}$ ) coupling with the inner core (real part), pointing out the need of iterations between SPs.

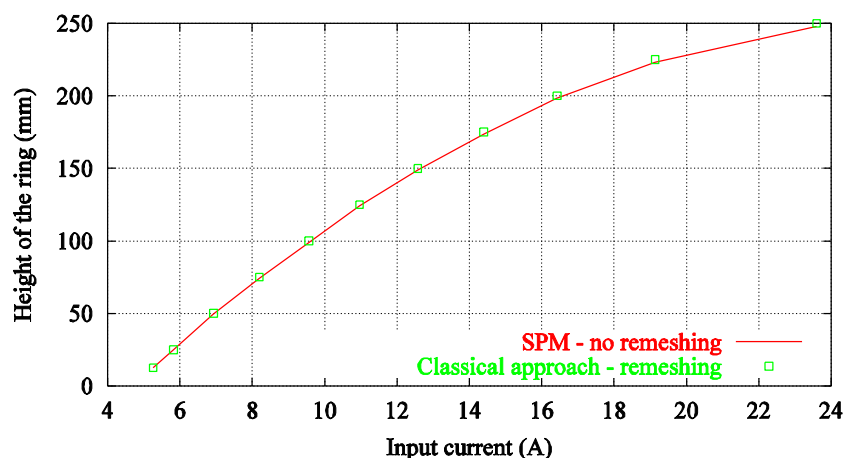

Fig. 3. Height of the ring versus input current $(50 \mathrm{~Hz})$.

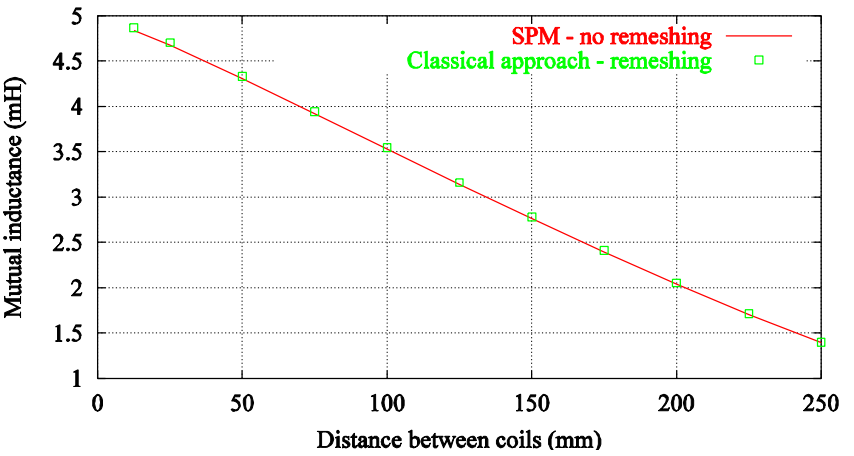

Fig. 4. Mutual inductance as a function of distance between the main coil and a search coil.

\section{CONCLUSIONS}

The developed SPM allows efficient parameterized FE studies of magnetic systems with changes of position and characteristics of conductors. Both massive and stranded conductors can be considered, with accurate calculations of skin and proximity effects, and thus of all the resulting quantities: Joule losses, forces, resistances, inductances, currents and voltages. The use of distinct meshes for the SP gives significant facilities. The method can naturally be extended to deal with moving systems, with non-linearities, which will be further developed.

\section{ACKNOWLEDGMENT}

This work is supported by the F.R.S.-FNRS (Belgium), the CNPq (Brazil) and the Belgian Science Policy (IAP P6/21).

\section{REFERENCES}

[1] Z. Badics et al., "An effective 3-D finite element scheme for computing electromagnetic field distorsions due to defects in eddy-current nondestructive evaluation," IEEE Trans. Magn., Vol. 33, No. 2, pp. 1012-1020, 1997.

[2] P. Dular, R. V. Sabariego, J. Gyselinck and L. Krähenbühl, "Subdomain finite element method for efficiently considering strong skin and proximity effects," COMPEL, vol. 26, no. 4, pp. 974-985, 2007.

[3] P. Dular, R. V. Sabariego, M. V. Ferreira da Luz, P. Kuo-Peng and L. Krähenbühl, "Perturbation Finite Element Method for Magnetic Model Refinement of Air Gaps and Leakage Fluxes," IEEE Trans. Magn., vol. 45, no. 3, pp. 1400-1403, 2009.

[4] P. Dular, R.V. Sabariego, M.V. Ferreira da Luz, P. Kuo-Peng and L. Krähenbühl, "Perturbation finite-element method for magnetic circuits," IET Science, Measurement \& Technology, vol. 2, no. 6, pp. 440-446, 2008.

[5] P. Dular, R.V. Sabariego and L. Krähenbühl, "Magnetic Model Refinement via a Perturbation Finite Element Method - From 1-D to 3D," COMPEL, vol. 28, no. 4, pp. 974-988, 2009.

[6] P. Dular, R. V. Sabariego, C. Geuzaine, M. V. Ferreira da Luz, P. KuoPeng and L. Krähenbühl, "Finite Element Magnetic Models via a Coupling of Subproblems of Lower Dimensions," IEEE Trans. Magn., vol. 46, no. 8, pp. 2827-2830, 2010.

[7] C. Geuzaine, B. Meys, F. Henrotte, P. Dular and W. Legros, "A Galerkin projection method for mixed finite elements," IEEE Trans. Magn., Vol. 35, No. 3, pp. 1438-1441, 1999.

[8] C. Golovanov, Y. Maréchal, and G. Meunier, "3D Edge Element based Formulation Coupled to Electric Circuits," IEEE Trans. Magn., vol. 34, no. 5, pp. 3162-3165, 1998.

[9] P. Dular, P. Kuo-Peng, C. Geuzaine, N. Sadowski and J.P.A. Bastos, "Dual Magnetodynamic Formulations and their Source Fields associated with massive and stranded inductors," IEEE Trans. Magn., vol. 36, no. 4, pp. 1293-1299, 2000.

[10] V.M. Machado, "Axisymmetric Eddy Current Problems Using a Special Hybrid FE/BE Method," IEEE Trans. Magn., vol. 39, no. 3, pp. 1143$1146,2003$. 